\title{
Research Paper \\ The Relationship Between Parenting Styles and Moral Development of Preschool Children Mediated by Children's Attachment
}

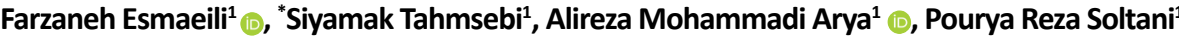

1. Department of Preschool Education, Faculty of Educational Sciences and Social Welfare, University of Social Welfare and Rehabilitation Sciences, Tehran, Iran

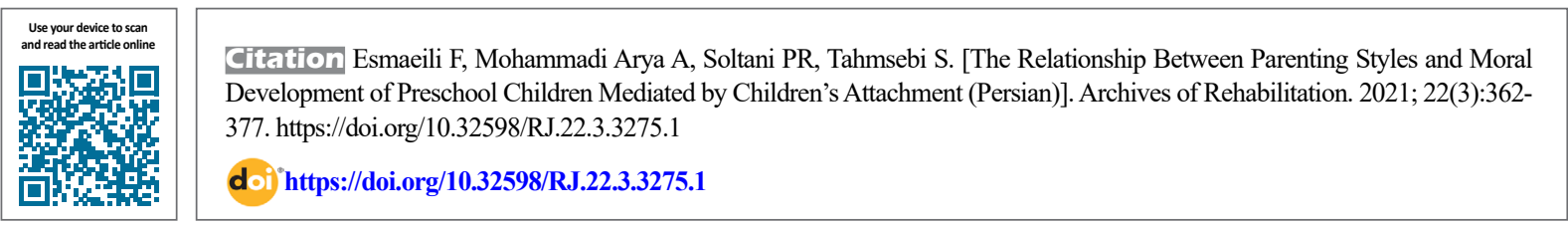

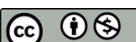

Received: 28 Nov 2020

Accepted: 11 Apr 2021

Available Online: 01 Oct 202
Keywords:

Parenting styles,

Moral development, Attachment, Children

\section{ABSTRACT}

Objective Family is like a school where parents teach the lessons of humanity and morality, which are the foundation of human happiness, to their children. Moral development is an essential part of children's understanding of moral rules and principles. The lack of moral development, along with anxiety, and depression exposes children to antisocial and suicidal behaviors. Moral development is associated with parenting styles and attachment. This study aims to investigate the relationship between parenting styles and moral development of preschool children mediated by children's attachment.

Materials \& Methods This descriptive correlational study was conducted on 128 children (64 girls and 64 boys) selected from 8 preschools in Tehran Province, Iran, in 2020 using a cluster sampling technique. The study data were collected using three questionnaires: Baumrind Parenting Styles Questionnaire (PSQ), the Kinship Center Attachment Questionnaire (KCAQ), and Lotfabadi Moral Development Questionnaire (MDQ). The first two tools were completed by mothers of children, while the MDQ was completed by children. The Kolmogorov-Simonov test, correlation test (Enter method), analysis of variance, and multiple regression analysis were used for data analysis in SPSS v. 21.

Results The regression analysis results showed that parenting styles had a significant relationship with moral development $\left(\mathrm{R}=0.74\right.$, Adjusted $\left.\mathrm{R}^{2}=0.54, \mathrm{P}=0.000\right)$. Also, attachment had a significant association with moral development $\left(R=0.71\right.$, Adjusted $\left.R^{2}=0.49, P=0.000\right)$. With one unit change in permissive, authoritarian, and authoritative parenting styles, the moral development of preschool children can be predicted by $43.5 \%, 19 \%$, and $37.6 \%$, respectively. Moreover, with one unit change in positive adjustment/ development, negative behavior, emotional reactivity, and distancing from caregiver support dimensions of attachment, the moral development of preschool children can be predicted by $33.6 \%, 38.4 \%$, $-26.4 \%$, and $-11.4 \%$, respectively. Also, the results of the Sobel test showed that the attachment variable was the mediator of the relationship of parenting styles with moral development $(z=2.29>1.96)$.

Conclusion The moral development of children is related to permissive, authoritarian, and authoritative parenting styles. Besides, children's attachment can mediate the relationship between parenting styles and moral development. More planning and follow-up are needed to improve the moral development of preschool children. When parents are consistent in their parenting styles, they provide their children with assertive and trusting behaviors. They should listen to their children and promote dialogue with them. Their discipline should be accompanied by warmness, logic, and flexibility and give their children the freedom to some extent. This treatment can help children develop their social behavior.

\section{${ }^{*}$ Corresponding Author:}

Siyamak Tahmsebi, PhD.

Address: Department of Preschool Education, University of Social Welfare and Rehabilitation Sciences, Tehran, Iran. Tel: +98 (937) 7318824

E-Mail: siyamak.tahmasebi@gmail.com 


\section{English Version}

\section{Introduction}

$\mathrm{T}$

he principles of humanity and morality are taught in the family. This education is crucial for children. Understanding morals are developed from infancy to adulthood [1]. Moral development is one of the critical issues in children to avoid committing crimes and behave ethically [2]. Today, people should pay attention to moral values more than ever because socio-political developments have caused traditional moral systems to lose their former effectiveness [3]. Achieving moral growth has consequences such as praising people [4]. Lack of moral development exposes children to anxiety and depression through antisocial and suicidal behaviors [5].

Parenting styles are the methods that parents use to raise their children. By these methods, they express their attitudes towards their children and the rules and standards that they apply to their children [6]. Parenting in different styles (authoritative, authoritarian, permissive) can affect children's personalities and behavior [7]. Authoritative parents control their children's behavior and have high expectations for puberty-based behavior. Authoritarian parents are very controlling and strict. Finally, permissive parents are loving parents who have no control over their children's behavior [8].

The child's desire to have intimate relationships with others and to feel more secure in their presence is called attachment [9]. The attachment between mother and child lays the foundation of a child's moral development in the future [10]. Therefore, parents and educators wish to raise moral children to have a healthy society [11]. According to Septani Mula et al., parents who create a secure attachment to their children and raise them in an authoritative parenting style produce children with social, emotional, and moral development [10]. Rahbar and Esmaeilpour studied primary school children in Jask Port, Iran. Their results showed that moral development had a significant negative relationship with authoritarian parenting style and a significant positive relationship with authoritative parenting style and secure attachment [12].

Although several studies have examined the relationship of parenting styles and attachment with children's moral development, very few studies have examined the mediating role of attachment. Therefore, the present study aims to investigate the relationship between parenting styles and moral development of preschool children mediated by children's attachment.

\section{Materials and Methods}

This research is a descriptive cross-sectional study conducted in 2019 for six months. The study population consisted of all preschool children (both genders) in Tehran City, Iran. Of whom, 128 children aged 6 years were selected from the schools in the north, west, east, and south of Tehran using the stratified sampling method. The inclusion criteria were willingness to participate in the study, having educated parents, and without mental health problems, while the exclusion criterion was returning incomplete questionnaires. Written informed consent was obtained from all subjects, and they were assured of the confidentiality of their information. The study was approved by the Ethics Committee of the University of Social Welfare and Rehabilitation Sciences (Code: IR.USWR.REC.1399.043).

The data collection tools were the Moral Development Questionnaire (MDQ) completed through interviews with the children, the Baumrind Parenting Styles Questionnaire (PSQ), and the Kinship Center Attachment Questionnaire (KCAQ), which were completed by mothers of children. The MDQ was developed by Lotfabadi to measure the moral development of children aged 5-11 years. It has 18 items and 6 subscales related to social and psychological factors of moral development. The face and content validities of this instrument were confirmed by experts, and its reliability was above 0.7 using the Cronbach $\alpha$ for the whole questionnaire [12]. The PSQ assesses Baumrind's permissive, authoritarian, and authoritative parenting styles. The reliability values of this questionnaire have been reported to be 0.69 for permissive, 0.77 for authoritarian, and 0.73 for authoritative dimensions [13]. Halpern and Copenberg developed the KCAQ to measure children's attachment in middle childhood (pre-primary and primary school) aged 3-12 years. The mother completed the questionnaire. The internal consistency of the KCAQ questionnaire is 0.85 , and its reliability based on the split-half method is 0.83 . Table 1 presents the Cronbach $\alpha$ and test-retest coefficients for total KCAQ and its four dimensions obtained for its Persian version in our study on 400 subjects [14]. The correlation between initial test and retest scores was significant $(\mathrm{P}=0.001)$.

The normality of quantitative data distribution was evaluated using the Kolmogorov-Smirnov test in SPSS v. 21. The data were described by descriptive statistics (Mean and Standard Deviation) and analyzed using the correlation test (Enter method), analysis of variance, and linear regression analysis, considering 0.05 as the significant level. 


\section{Results}

The study participants included 64 girls $(50 \%)$ and 64 boys $(64 ; 50 \%)$. The educational level of children's fathers and mothers mainly was diploma $(n=62,48.4 \%$ for fathers; $\mathrm{n}=61,47.7 \%$ for mothers), and the lowest frequency was related to the Master's degree ( $n=8,6.3 \%$ for fathers; $n=3$, $2.3 \%$ for mothers). As shown in Table 2, the mean total scores for the variables of parenting styles, moral development, and attachment were high.

The Kolmogorov-Smirnov test results showed that data were normally distributed according to skewness and kurtosis values (Table 3). The results of regression analysis (Table 4) showed the significant relationship of parenting styles with moral development $\left(\mathrm{R}=0.74\right.$, Adjusted $\mathrm{R}^{2}=0.54$, $\mathrm{P}=0.000)$ and the relationship of attachment with moral de- velopment $\left(\mathrm{R}=0.71\right.$, Adjusted $\left.\mathrm{R}^{2}=0.49, \mathrm{P}=0.000\right)$. Parenting methods, intimate upbringing, and discussion of moral issues help children understand morality. Children whose parents listen to them wholeheartedly ask enlightening questions, confront high-level reasoning, and make great strides in moral development. In contrast, children whose parents use threats or harsh statements change little or stop at the early stages of moral development.

According to the results in Table 5, with one unit change in permissive, authoritarian, and authoritative parenting styles, the moral development of preschool children can be predicted by $43.5 \%, 19 \%$, and $37.6 \%$, respectively. Moreover, with one unit change in positive adjustment/development, negative behavior, emotional reactivity, and distancing from caregiver support dimensions of attachment, the moral development of preschool children can be predicted by $33.6 \%$,

Table 1. Reliability coefficients of cronbach $\alpha$ and test-retest for the dimensions of the kinship center attachment questionnaire

\begin{tabular}{ccccc}
\hline Dimensions & \multicolumn{2}{c}{ Boys } & \multicolumn{2}{c}{ Girls } \\
\cline { 2 - 5 } & Alpha & Test-Retest & Alpha & Test-Retest \\
\hline Positive adjustment/development & 0.78 & 0.73 & 0.83 & 0.89 \\
\hline Negative behavior & 0.71 & 0.88 & 0.77 & 0.90 \\
\hline Emotional reactivity & 0.72 & 0.85 & 0.79 & 0.84 \\
\hline Distancing from caregiver support & 0.85 & 0.91 & 0.71 & 0.94 \\
\hline Total & 0.73 & 0.81 & 0.78 & 0.86 \\
\hline
\end{tabular}

Table 2. Mean scores of the study variables

\begin{tabular}{ccccc}
\hline & \multicolumn{1}{l}{ Variables } & Mean \pm SD & Min. & Max. \\
\hline & Positive adjustment/development & $23.3516 \pm 4.15$ & 13.00 & 30.00 \\
Negative behavior & $15.5313 \pm 2.75$ & 8.00 & 20.00 \\
KCAQ & Emotional reactivity & $24.3281 \pm 3.16$ & 30.00 & 20.00 \\
& Distancing from caregiver support & $16.2891 \pm 2.16$ & 15.00 & 97.00 \\
TSQ & Total & $79.4453 \pm 7.60$ & 58.00 & 49.00 \\
& Permissive & $39.5234 \pm 4.88$ & 25.00 & 46.00 \\
Authoritarian & $40.6250 \pm 2.66$ & 35.00 & 49.00 \\
Authoritative & $39.3594 \pm 4.90$ & 26.00 & 125.00 & 232.00 \\
\hline
\end{tabular}

KCAQ: The Kinship Center Attachment Questionnaire; PSQ: Parenting Styles Questionnaire; MDQ: Moral Development Questionnaire. 
Table 3. Skewness and kurtosis values for testing the normality of data distribution

\begin{tabular}{ccc}
\hline Variable & Skewness & Kurtosis \\
\hline KCAQ & -0.569 & 2.007 \\
PSQ & -0.901 & 1.234 \\
MDQ & -2.047 & 2.165 \\
\hline
\end{tabular}

KCAQ: The Kinship Center Attachment Questionnaire; PSQ: Parenting Styles Questionnaire; MDQ: Moral Development Questionnaire.

$38.4 \%,-26.4 \%$, and $-11.4 \%$, respectively. Children with authoritative parents are more psychologically and socially competent than their peers with authoritarian or permissive parents. These children are responsible, adaptive, creative, curious, and confident. According to psychologists, the authoritative parenting style is the most efficient method; children of authoritative parents have more social skills and selfesteem than those of authoritarian or permissive parents. According to the results of the Sobel test in Table 6, it was found that the variable of attachment styles is a mediating variable. The $\mathrm{z}$ value was 2.96 , greater than 1.96 , indicating the effect of children's attachment on the relationship between parenting styles and moral development.

\section{Discussion and Conclusion}

This study aimed to determine the mediating role of preschool children's attachment in the relationship between parenting styles and moral development. The level of authoritarian parenting style was different according to gender, whereas women's attitudes toward this style were higher. Distancing from caregiver support as one of the attachment components was different in terms of gender, where the girls had higher scores. The mean scores of parenting style, attachment, and moral development were also different in terms of parents' education, but it was not statistically significant.

The moral development of children had a significant positive relationship with permissive, authoritarian, and authoritative parenting styles $(\mathrm{P}=0.001)$. This finding is consistent with the results of Rahbar and Salaripour [17], Babajanzadeh and Otaghsara [19], Morvati et al. [23]. Zokaei's study found no significant relationship between authoritarian parenting style and social development [18]. In our study, there was also a significant relationship between children's moral development and attachment. This result is consistent with the results of Shahri [24], Pour Movadat [25], and Safaee [26]. In Septani et al.'s study, a family with four children was selected, and its authoritative parenting method and secure attachment were assessed. In contrast, in our study, 128 children and their mothers participated, and the children's attachment was measured as a mediating variable. The results regarding the mediating role of children's attachment in the relationship between parenting styles and moral development were consistent with the results of Ghorbani [27] and Peymannia [28].

When parents are consistent in their parenting styles, they provide their children with assertive and trusting behaviors; they listen to their children and promote dialogue with them. Their discipline is accompanied by warmness, logic, and flexibility, and they give their children freedom to some extent. This treatment helps children develop their social behavior [17]. Authoritarian style is an inefficient method that can cause various emotional, moral, and behavioral problems in children [15]. However, children of authoritative parents show positive social behaviors such as cooperation, love, empathy, etc. A healthy parenting style can predict children's social and moral characteristics [3]. Parents are

Table 4. Results of regression analysis to examine the relationship of parenting styles and attachment with moral development in children

\begin{tabular}{ccccccc}
\hline Model & $\mathbf{R}$ & $\mathbf{R}^{\mathbf{2}}$ & Adjusted $\mathbf{R}^{\mathbf{2}}$ & Std. Error & $\mathbf{F}$ & Sig. \\
\hline PSQ - MDQ & 0.746 & 0.557 & 0.547 & 12.89188 & 52.0 & 0.000 \\
KCAQ - MDQ & 0.713 & 0.509 & 0.493 & 13.63205 & 31.86 & 0.000 \\
\hline & & & & & $\begin{array}{c}\text { Archives of } \\
\text { Rehabilitation }\end{array}$
\end{tabular}

KCAQ: The Kinship Center Attachment Questionnaire; PSQ: Parenting Styles Questionnaire; MDQ: Moral Development Questionnaire. 
Table 5. Regression coefficients

\begin{tabular}{|c|c|c|c|c|c|c|}
\hline & Model & B & Std. Error & Beta & $\mathbf{t}$ & Sig. \\
\hline \multirow{5}{*}{ PSQ } & Constant & 78.254 & 18.156 & \multirow[b]{2}{*}{0.435} & 4.310 & 0.000 \\
\hline & Permissive & 1.704 & 0.349 & & 4.883 & 0.000 \\
\hline & & & & & & \\
\hline & Authoritarian & 1.365 & 0.628 & 0.190 & 2.173 & 0.032 \\
\hline & Authoritative & 1.467 & 0.350 & 0.376 & 4.197 & 0.000 \\
\hline \multirow{6}{*}{ KCAQ } & Constant & 123.720 & 14.595 & \multirow{3}{*}{0.336} & 8.477 & 0.000 \\
\hline & & & & & & \\
\hline & Positive adjustment/development & 1.551 & 0.489 & & 3.172 & 0.002 \\
\hline & Negative behavior & 2.671 & 0.738 & 0.384 & 3.619 & 0.000 \\
\hline & Emotional reactivity & -1.599 & 0.521 & -0.264 & -3.066 & 0.003 \\
\hline & Distancing from caregiver support & -1.011 & 0.785 & -0.114 & -2.289 & 08000 \\
\hline
\end{tabular}

KCAQ: The Kinship Center Attachment Questionnaire; PSQ: Parenting Styles Questionnaire; MDQ: Moral Development Questionnaire.

the source of children's social experiences due to their emotional dependence on them, which ultimately leads to the formation of moral values during childhood and adulthood. The formation of secure attachment seems to be the basis of a healthy moral orientation. Secure attachment develops in childhood when parents respond to the children's needs. The children can explore the environment with a sense of security and confidence to confront challenges. According to attachment theory, the initial interaction of a child with a caregiver takes the form of internal beliefs. The effects of attachment in childhood extend to adulthood in the areas of moral development and close relationships. This study had some limitations, including the lack of similar studies in Iran and other countries, a high number of questions that made children tired, and the lack of cooperation of some mothers in completing questionnaires.

Parents use various parenting styles in dealing with their children, but the predominant style is the permissive style. In addition to the direct effect, children's attachment is indirectly related to their moral development. Moral develop- ment is formed in the safe context of the family and during the communication and emotional interaction of parents with children; thus, children direct their behaviors accordingly. Insecure, avoidant, and ambivalent people are underprivileged due to deviation from the main path of the attachment system. The results of this study can provide important information to parents, teachers, and educators. If parents pay attention to their children's needs with more kindness and sensitiveness, the children's attachment can be more secure. Since secure attachment develops in childhood, the moral development of adolescents and youth depends on their attachment in childhood. For parents who raise their children with permissive and authoritative styles, teaching parenting skills and familiarity with parenting techniques can help them promote their children's moral development. They should be reminded that the child's psychological and cultural personality is established before the age of 6 when the child spends more time in the family.

Table 6. Results of sobel test for examining the mediating role of attachment in the relationship between parenting styles and moral development

\begin{tabular}{cccccccc}
\hline Independent variable & Dependent variable & $\mathbf{B}$ & $\boldsymbol{\beta}$ & Std. Error & $\mathbf{R}$ & $\mathbf{R}^{2}$ & Sig. \\
\hline Parenting styles & Attachment & 0.60 & 0.87 & 0.030 & 0.87 & 0.76 & 0.000 \\
Attachment & Moral development & 1.32 & 0.49 & 0.211 & 0.49 & 0.24 & 0.000 \\
\hline & & & & & & & $\begin{array}{c}\text { Archives of } \\
\text { Rehabilitation }\end{array}$
\end{tabular}

KCAQ: The Kinship Center Attachment Questionnaire; PSQ: Parenting Styles Questionnaire; MDQ: Moral Development Questionnaire. 


\section{Ethical Considerations}

Compliance with ethical guidelines

This study was approved by the Ethics Committee of the University of Social Welfare and Rehabilitation Sciences (Code: IR.USWR.REC.1399.043). All ethical principles are considered in this article. The participants were informed about the purpose of the research and its implementation stages. They were also assured about the confidentiality of their information. They were free to leave the study whenever they wished, and if desired, the research results would be available to them.

\section{Funding}

The paper was extracted from the MA. thesis of the first author at the Department of Preschool Education, Faculty of Educational Sciences and Social Welfare, University of Social Welfare and Rehabilitation Sciences, Tehran.

\section{Authors' contributions}

Conceptualization: Farzaneh Ismaili, Siamak Tahmasebi, Alireza Mohammadi Aria; Research: Pouria Reza Soltani; Editing and finalizing: Farzaneh Ismaili and Siamak Tahmasebi.

\section{Conflict of interest}

The authors declared no conflict of interest.

\section{Acknowledgments}

The authors would like to thank the Education Organization of Tehran Province and the parents of children who participated in this study. 
This Page Intentionally Left Blank 


\section{مقاله يزوهشى بررسي ارثباط بين سبكهاي فرزنديرورى با رشد اخلاقي كودكان ييش از دبستان با ميانجي كَّى دلبستيَي كودى}

فرزانه اسمعيلى 'ه. "سيامك طهماسبى'، عليرضا محمدى آريا' هـ يوريا رضاسلطانى'

1. كروه بيش دبسناني، دانشكده علوم تربيتي و رفاه اجتماعي، دانشكاه علوم توائبششي و سلامت اجتماعي، تهبران، ايران.

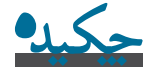

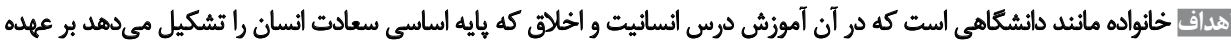

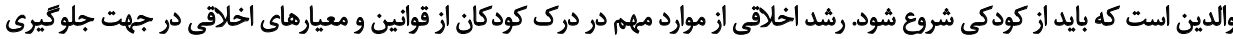

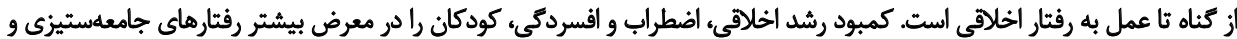

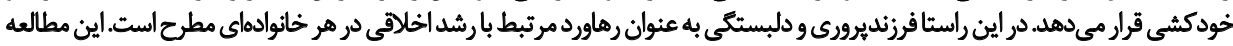

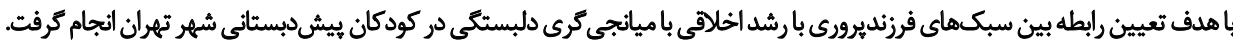

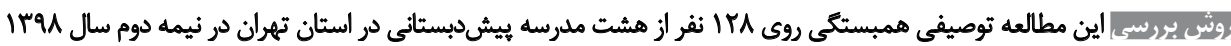

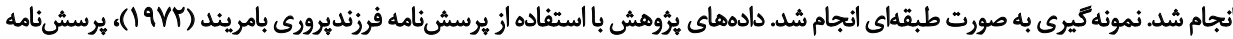

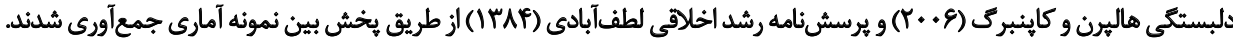

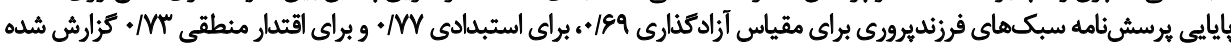

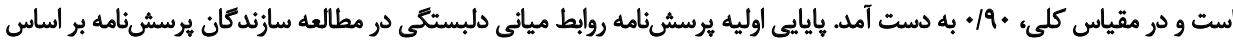

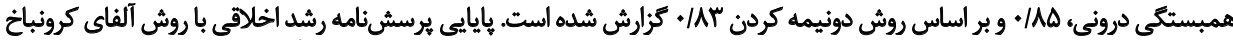

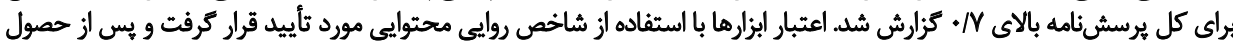

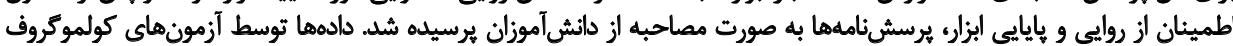

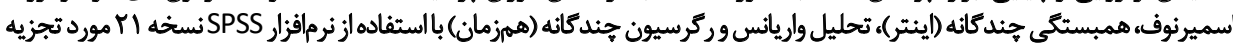
وتحليل قرار ترفتند.

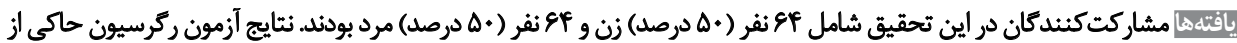

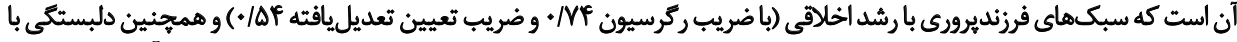

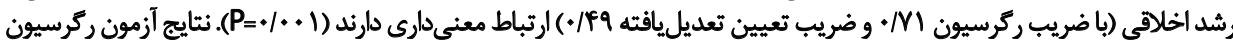

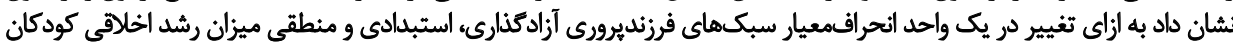

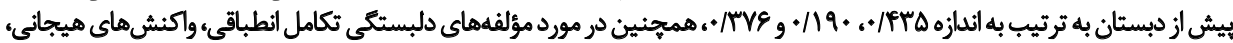

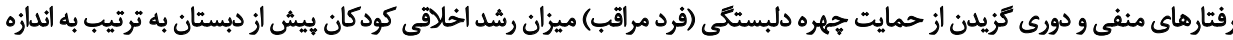

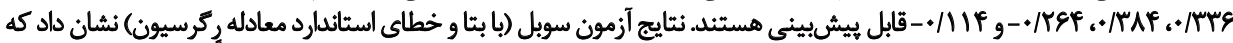

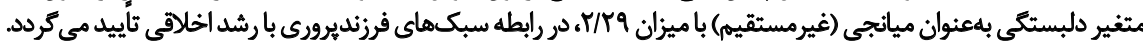

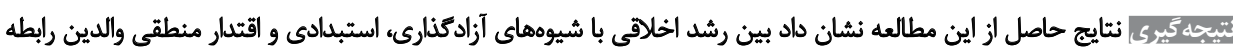

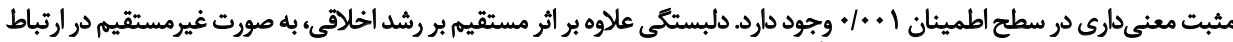

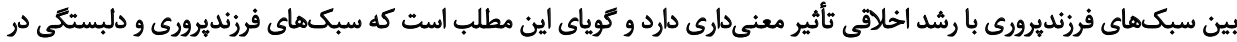

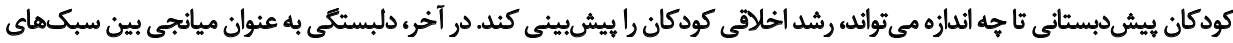

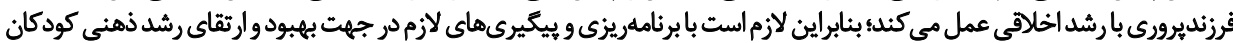

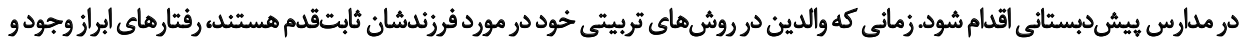

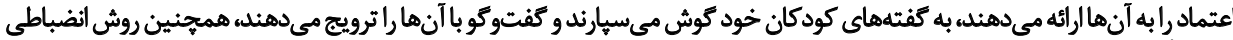

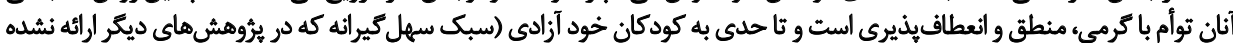

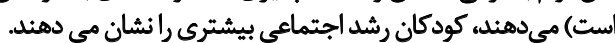

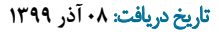

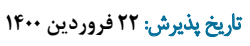

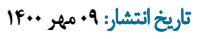

كليدوأرهها: سبكهاى فرزنديرورى، رشد اخلاقى، دلبستئكى، بيشدبستانى، كودى

$$
\begin{aligned}
& \text { "نويسينده مسئول: } \\
& \text { دكترسيامك طهماسبي }
\end{aligned}
$$

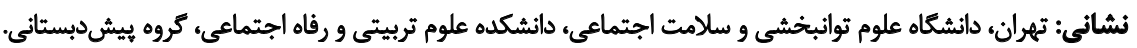

$$
\begin{aligned}
& \text { تلفن: } \\
& \text { راياثامه: siyamak.tahmasebi@gmail.com }
\end{aligned}
$$


و سرزنش آنها را فراهم نياورند [9]. سبكهاى فرزنديرورى،

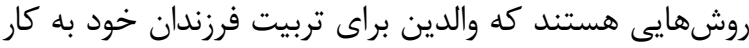

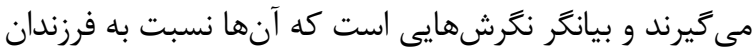

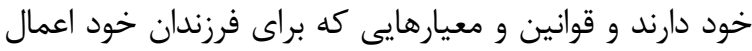

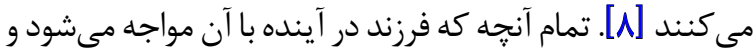

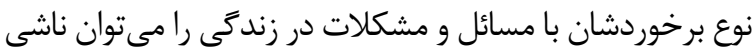

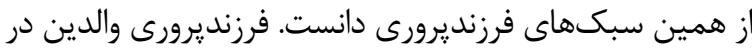

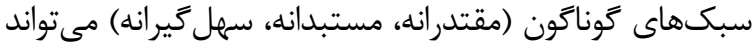

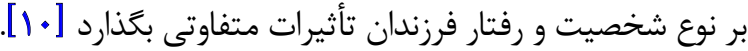

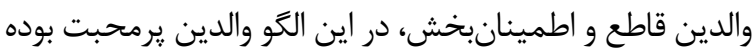

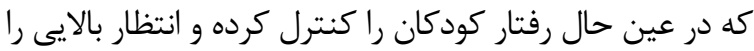

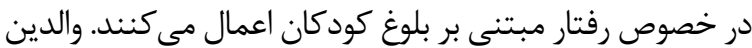

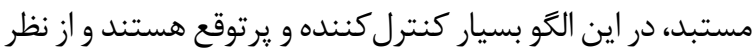

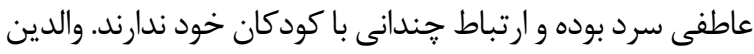

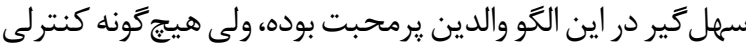
بر رفتار كودكان اعمال نمىكنيند [11]

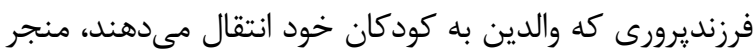

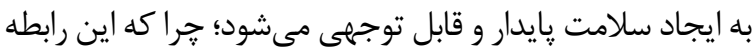

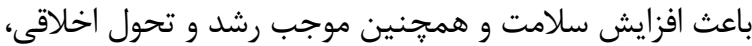

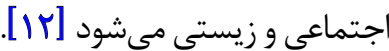

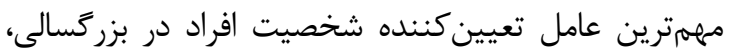

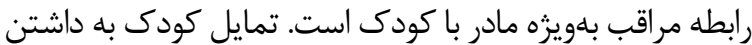

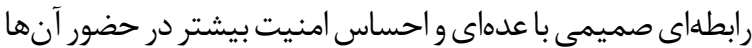

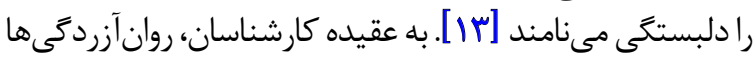

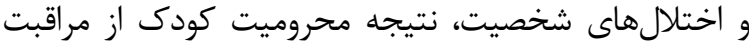

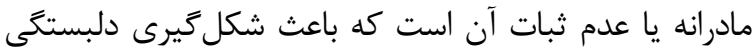

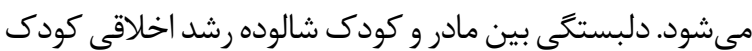

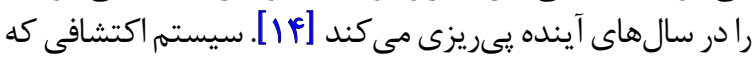

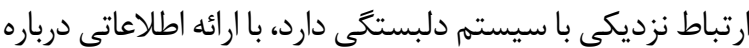

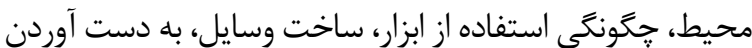

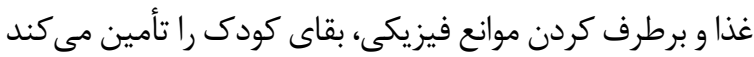

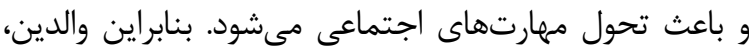

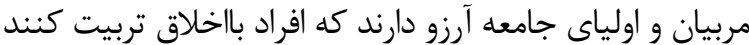

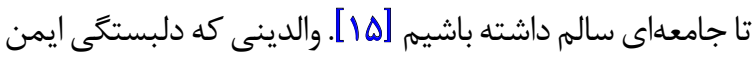

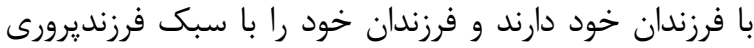

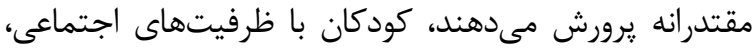

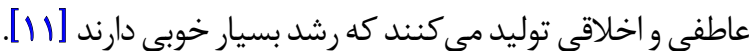

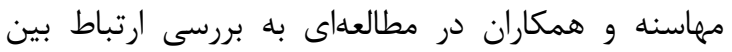

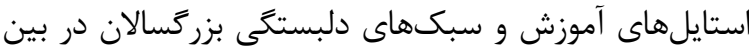

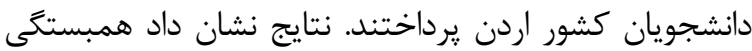

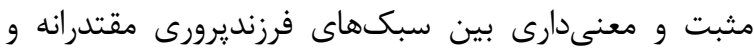

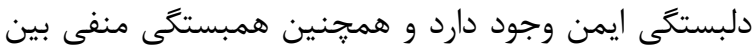

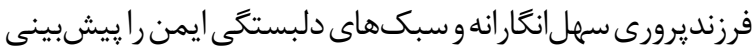

مقدمه

خانواده مانند دانشكاهى است كه در آن درس انسانيت و

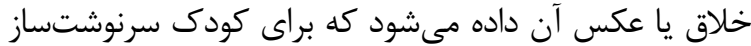

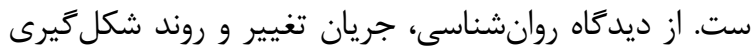

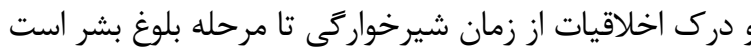

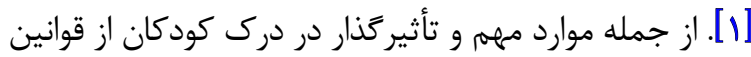

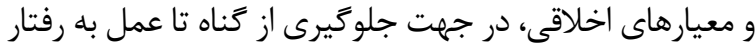

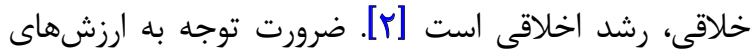

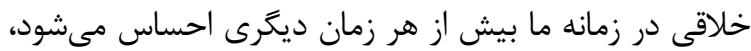

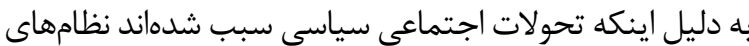

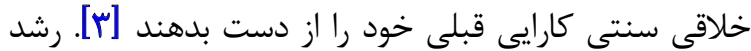

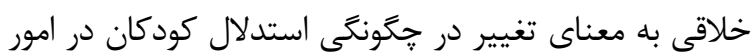

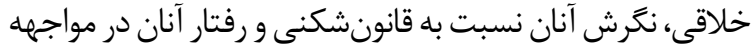

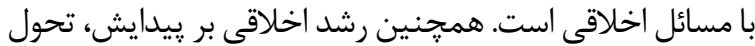

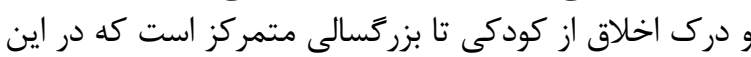

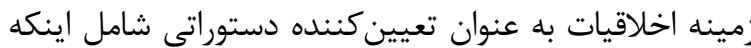

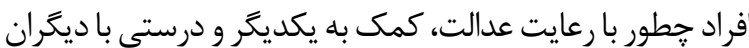

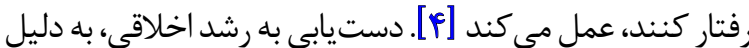

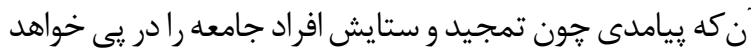

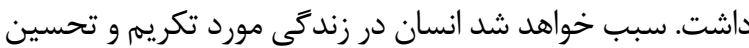

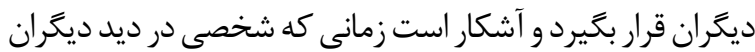

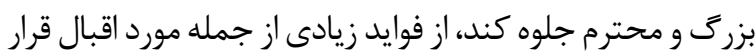

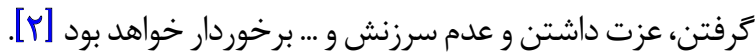
جان لاك معتقد است كودى وقتى متولد مىشود، ذهن او

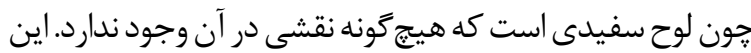

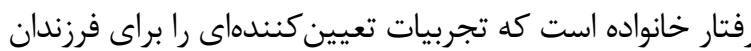

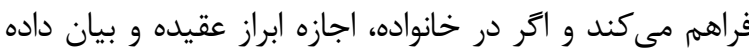

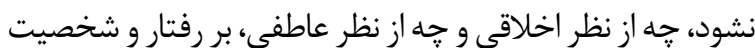

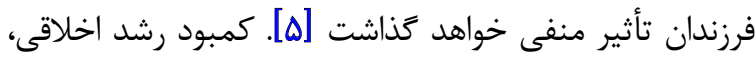

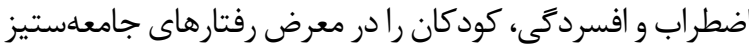

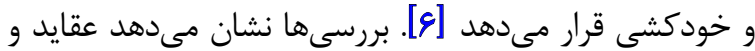

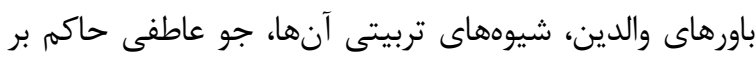

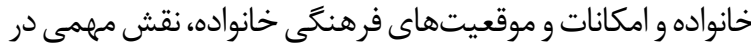

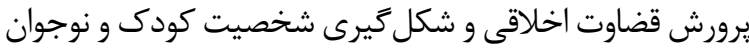

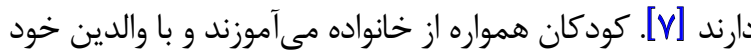

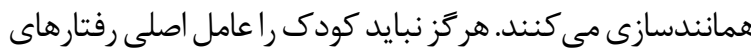

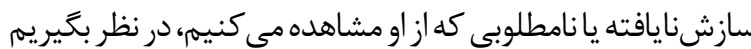

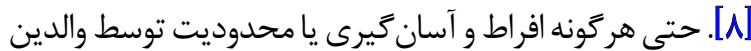
عواقب ناخوشايندى براى كودكان به ديه دنبال دارد و و ممكن است است

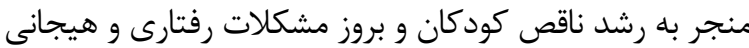

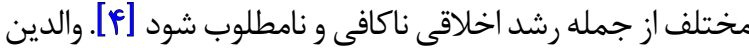

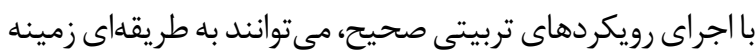

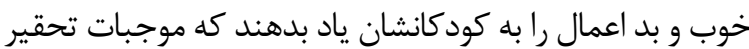


نمونه كيرى طبقهاى انتخاب شد. معيار هاى ورود به مطالعه شامل

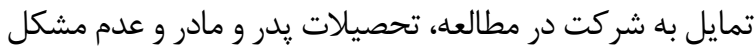

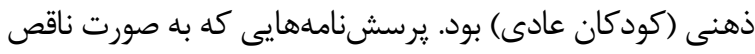

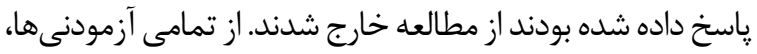

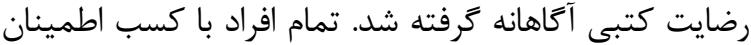

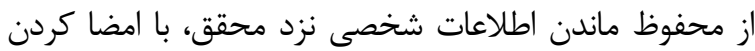

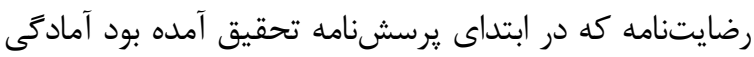

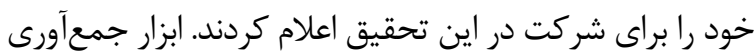

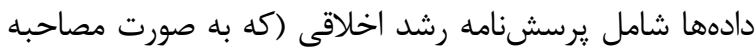

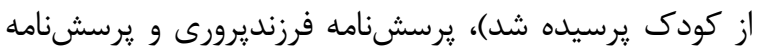

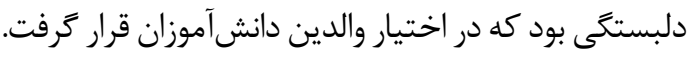

$$
\text { يرسش نامه شيوههاى فرزنديرورى بامريند }
$$

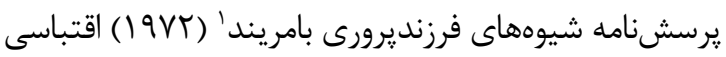

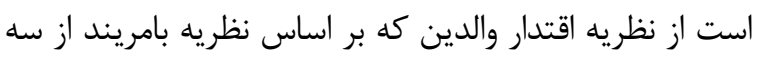

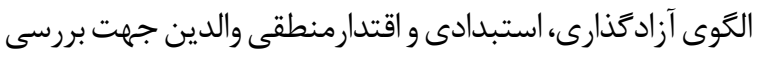

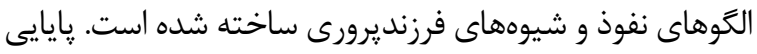

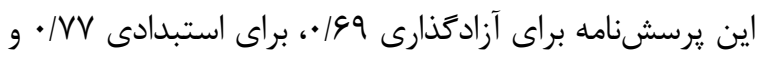

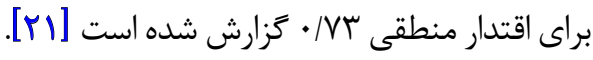

يرسش نامه روابط ميانى دلبستخى (KCAQ)

يرسشنامه روابط ميانى دلبستخى توسط هاليرن و كاينبرگ

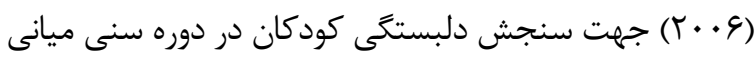

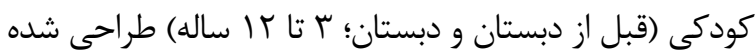

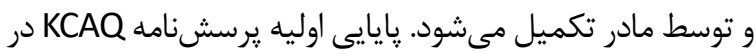

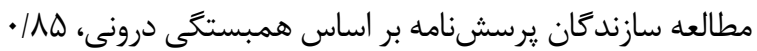

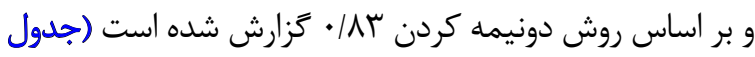

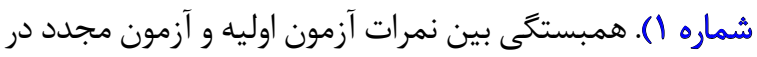

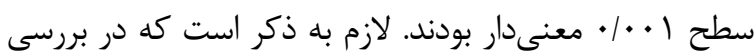

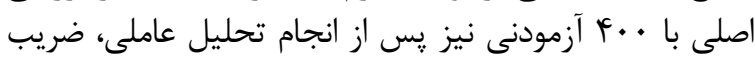

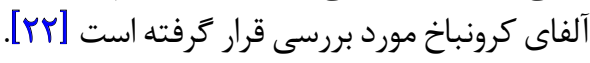

$$
\text { يرسشنامه رشد اخلاقى }
$$

يرسشنامه رشد اخلاقى كودكان توسط دكتر لطفآبادى

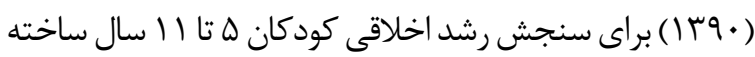

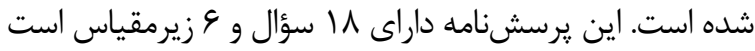

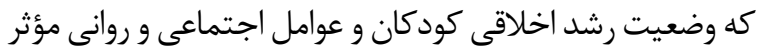

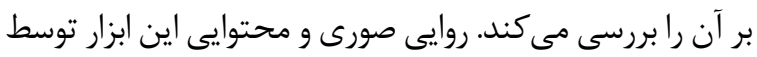

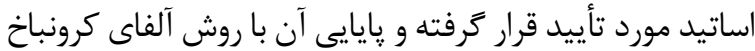

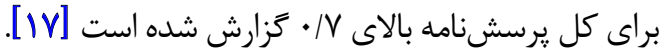
ابتدا نرمال بودن توزيع دادههاى كمى با استفاده از آزمون

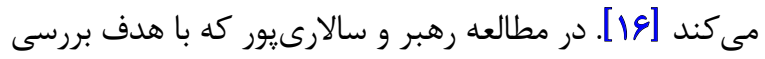

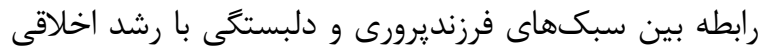

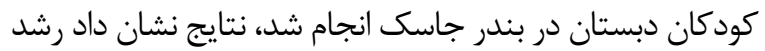

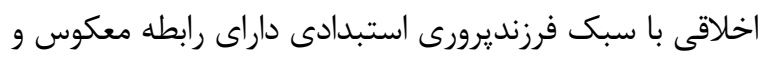

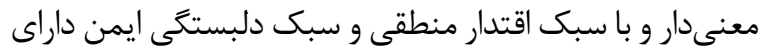

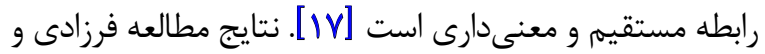

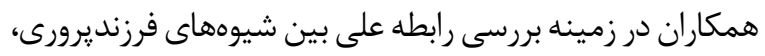

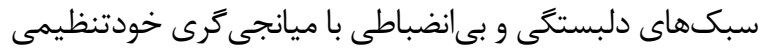

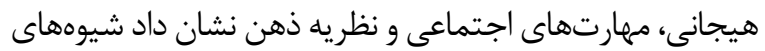

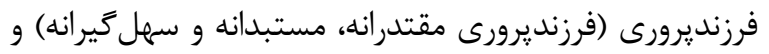

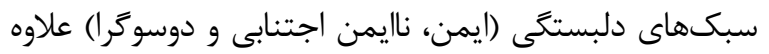

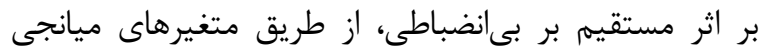

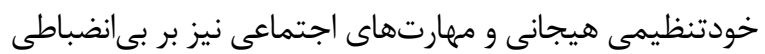

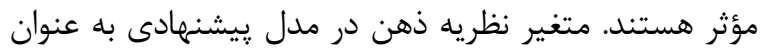

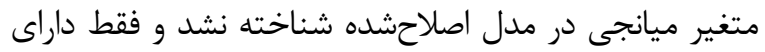

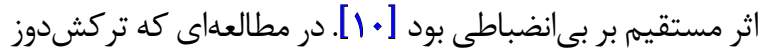

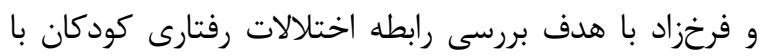

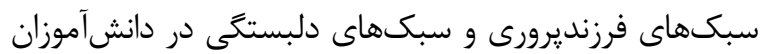

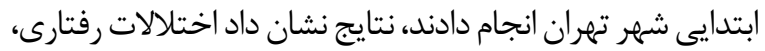

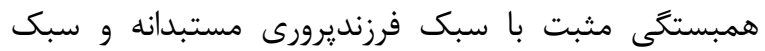

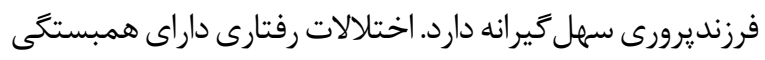

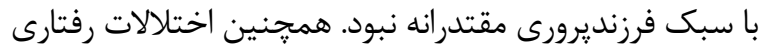

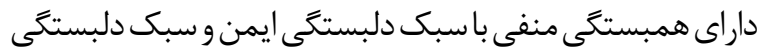

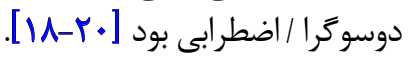

اخرجه مطالعات متعددى به بررسى رابطه بين سبكهاى

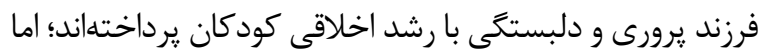

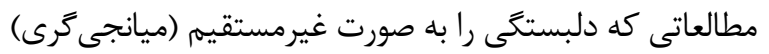
بررسى نمايد، بسيار اندك است؛ بنابر ائه

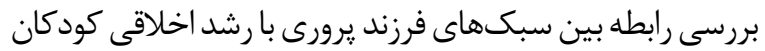

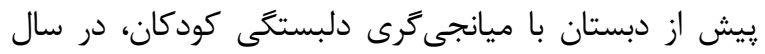

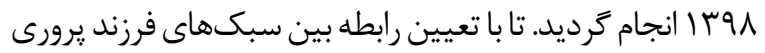

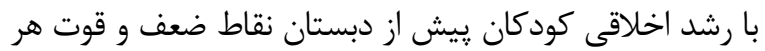

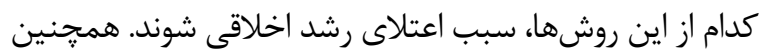

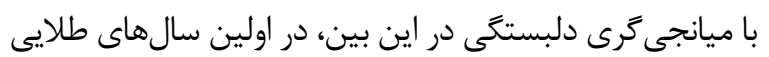

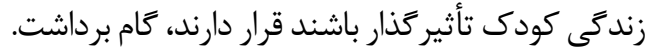

$$
\text { روش بررسى }
$$

اين يزوهش از نوع توصيفى بود كه به صورت مقطعى طى سال

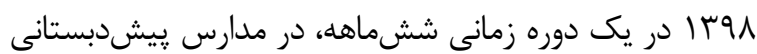

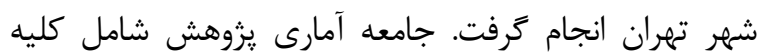

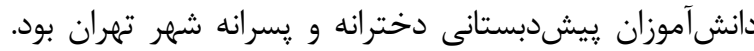

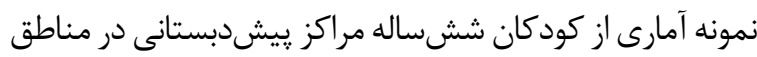

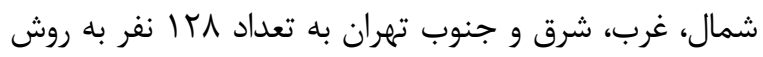

\section{Bamerind}


جدول ا. ضرايب يايايى به روش آلفاي كرونباخ و بازآزمايى براي مؤلفههاي يرسشنامه (KCAQ)

\begin{tabular}{|c|c|c|c|c|}
\hline \multicolumn{2}{|c|}{ 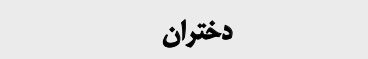 } & \multicolumn{2}{|c|}{ ئسران } & \multirow{2}{*}{ مؤلفههاى يروششنامه } \\
\hline ضريب بازازمايى & ضريب ألفا & ضريب بازأزمايى & صريب ألفا & \\
\hline.$/ 19$ & . AM & $\cdot M$ & $\cdot / \mathrm{r}$ & تكامل انطباقى مثبت \\
\hline.$/ 9$. &.$/ W$ &.$/ M$ & $\cdot / M$ & رفتارهاى منفى \\
\hline - /Ar &.$/ 19$ & . /NA & $\cdot / r$ & واكنش هاى هيجانى \\
\hline.$/ 94$ & $\cdot M$ &.$/ 91$ & . INA & دورى كزيلن از حمايت جهره دلبستكى \\
\hline $\mathscr{I N}$ &.$/ \mathrm{V} \Lambda$ &.$|A|$ &.$/ M^{2}$ & نمره كل \\
\hline
\end{tabular}

بهدستآمده براى متغيرهاى سبكهاى فرزنديرورى و رشد

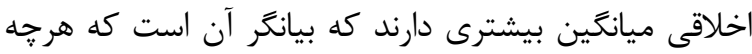

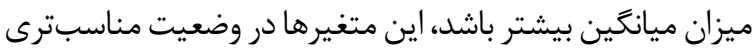

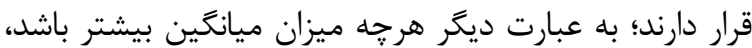

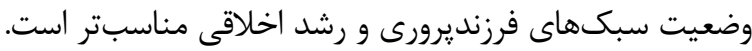

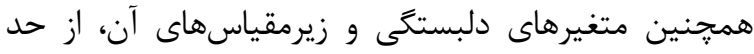

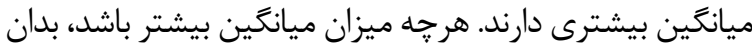
معنى است كه اين متغيرها در وضعيت مناسبترين دئرى قرار دارند. با توجه به جدول شماره با، متغيرهاى مورد نظر داراى

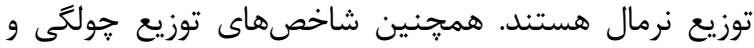

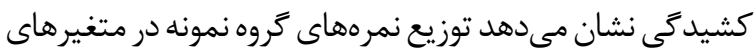

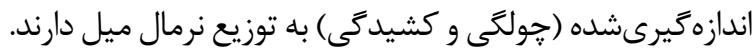
نتايج بهدستآمده از آزمون ركرسيون در جدول شماره F حاكى

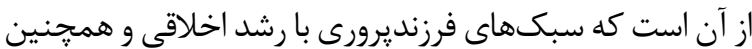

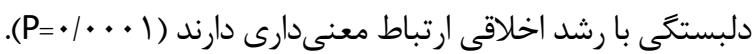

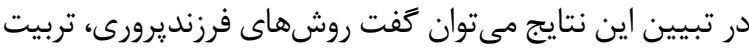

كولموگروف اسميرنوف توسط نرمافزار SPSS نسخه ال بررسى شد و دادهها با روشهاى آمار توصيفى (فراوانى و فراوانى نسبى)،

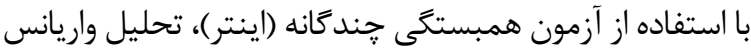

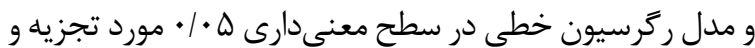
تحليل آمارى قرار گرفتند.

يافتهها

آمار توصيفى ويزگ گیى هاى جمعيتشناختى نمونه تحقيق نشان

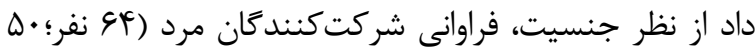

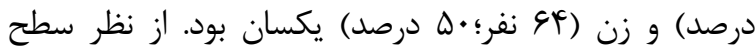

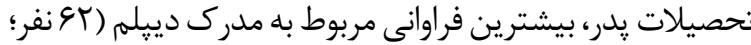

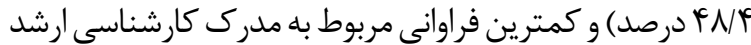

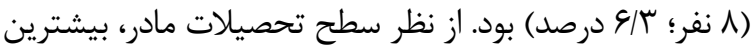

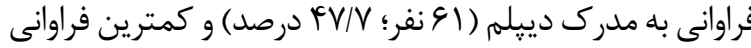

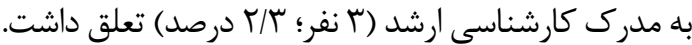
بر اساس يافتههاى جدول شيماره Y، ميانگين نمره كل

جدول ץ. آمارههاى توصيفى متغيرهاي دلبستكى، سبكهاى فرزنديرورى و رشد اخلاقى

\begin{tabular}{|c|c|c|c|c|}
\hline بيشترين & كمترين & مياتكين+نانحرافمعيار & مؤلفهها & متغيرها \\
\hline$r * \%$ & $1 \% \%$ & $\Gamma / T \Delta \mid \& \pm F / 1 \Delta$ & تكامل انطباقى مثبت & \\
\hline$r . \%$ & N.. & $\mid Q / \Delta T \pm T / N \Delta$ & واكتش هيجانى & \\
\hline$r \%$ & $10 / \%$ & $M / M T \pm M / 18$ & رفْتارهاى منفىى & ملبستكى \\
\hline$r . \%$ & $1 \%$ & $\mid E / Y \lambda \pm T / 18$ & دورى كزيلن از حمايت جهره دلبستكى / فرد مراقب & \\
\hline $9 \vee / *+$ & $\Delta N \cdot$. & $\mathrm{Vq} / \mathrm{ft} \pm \mathrm{V} / \mathrm{g}+$ & 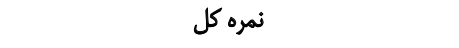 & \\
\hline$P q / .$. & $r \Delta /$. & $r q / \Delta T \pm F / M$ & شيوه آزادكنارى & \\
\hline$+\% 1 .$. & $r \Delta / .$. & $\mathrm{H} / \mathrm{AT} \pm \mathrm{Y} / \mathrm{ES}$ & شيوه استبدادى & سبك هاي فرزثلديوروى \\
\hline$p q / .$. & rel.o. & $m / T^{m} \Delta \pm F / q$. & شيوه اقتّلدار منطقى & \\
\hline 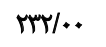 & $\mid r \Delta / \omega$ & $194 / * \Delta \pm 1 \% / 19$ & رشد اخلاقى & رشهد اخلاقى \\
\hline
\end{tabular}


جدول "ا. نتايج ئرمال بودن توزيع دادهها

\begin{tabular}{|c|c|c|}
\hline كثيدمى & هولثيى & متغير \\
\hline$Y / . . V$ & $-.10<9$ & للبستكى \\
\hline $1 / m$ & $-+/ q+1$ & فرزنديرورى \\
\hline$r / I F Q$ & $-Y / \cdot P Y$ & رشد اخلاقي \\
\hline
\end{tabular}

توانبخنتى

مستقيم مبتنى است. اثر غيرمستقيم ab يك بر آورد خاص نمونه

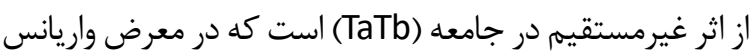

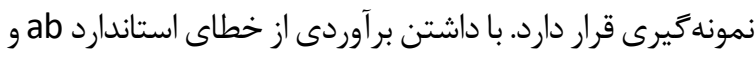
با فرض نرمال بودن دادهها و توزيع نمونه

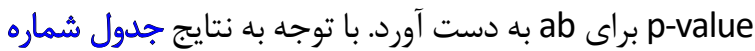

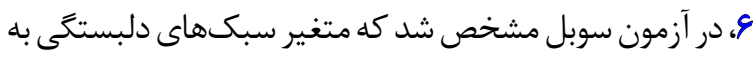

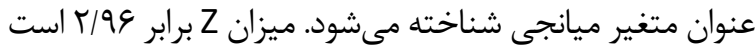

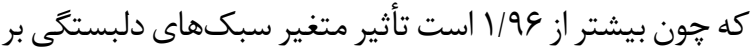

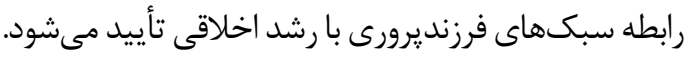

ب

اين مطالعه با هدف تعيين رابطه سبكهاى فرزنديرورى با

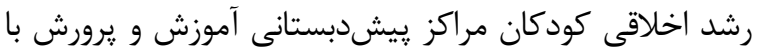

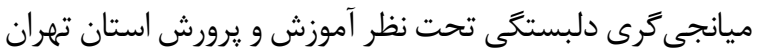

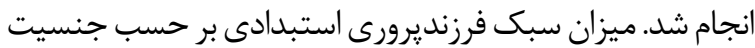

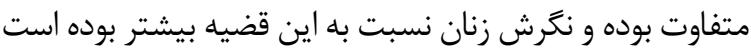

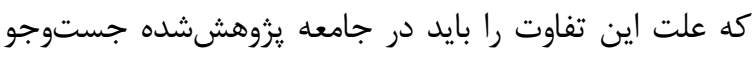

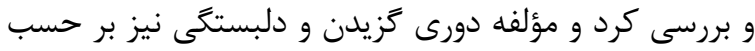

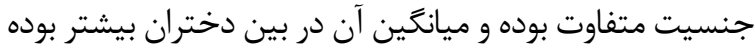

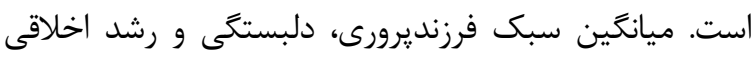

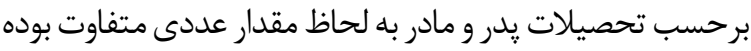

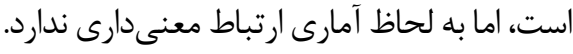
با توجه به نتايج، بين نمره رشد اخلاقى باشيوههاى آزادگذارى،

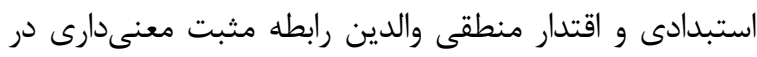

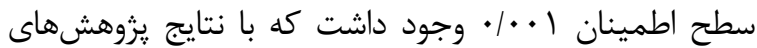

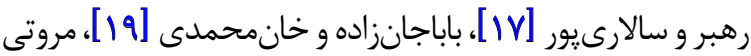

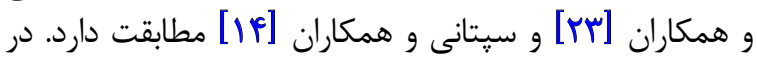

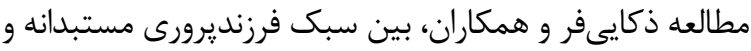

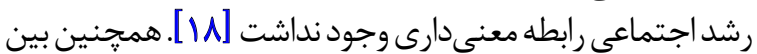

صميمانه و بحث درباره مسائل اخلاقى، به درك اخلاقى در

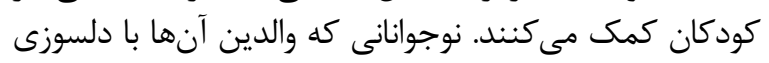

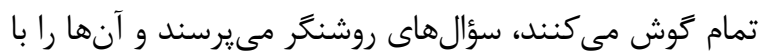

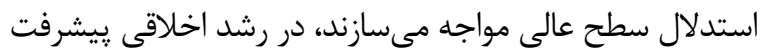

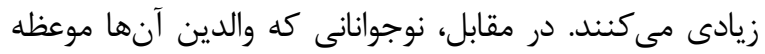

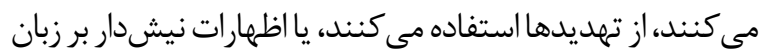

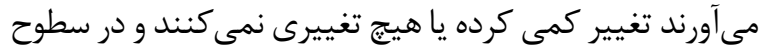
ابتدايى مراحل رشد اخلاقى متوقف مىشوند.

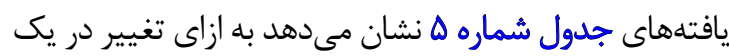

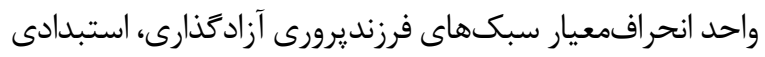

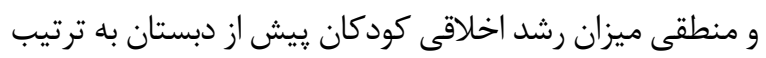

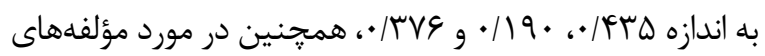

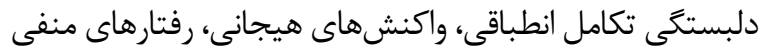

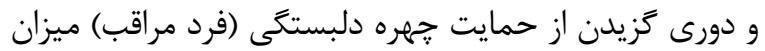

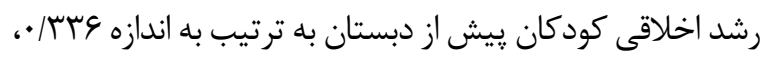

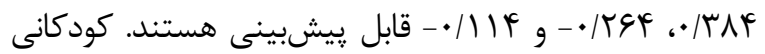

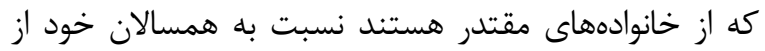

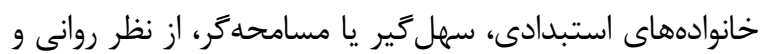

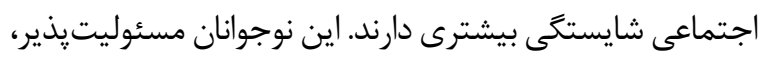

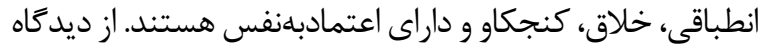

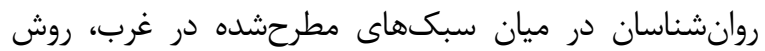

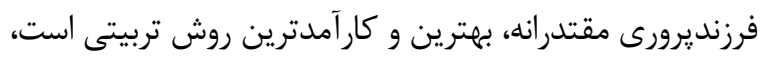

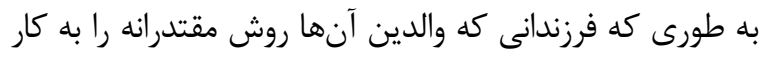

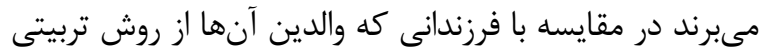

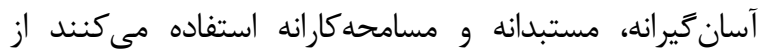
مهارتهاى اجتماعى و عزت نفس بيشترى برخورانه به كودكان و نوجوانان ديخر اميدوارترند. آزمون سوبل براى انجام استنباط در مورد ضريب اثر

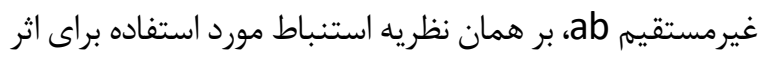

جدول ع. ارتباط بين سبكهاى فرزنديروري و دلبستكى با رشد اخلاقى

\begin{tabular}{|c|c|c|c|c|c|c|}
\hline سطح معنى فارى & تحليل وأريانس & خطاى معيار & ضريب تعيين تعديليافته & ضريب تعيين & ضريب همبستكى هند كانه & مثغير \\
\hline .10 .01 & $\Delta r / \cdot T$ & IY/A9IM & . /AFY &.$/ \Delta \Delta V$ & Mes & فرزنديرورى با رشد اخلاقى \\
\hline$\circ 1 \ldots 1$ & MVIN & $\mid M / g \pi t+0$ &.$/ 4 q$ & .10 .9 & . MIr & دلبستكى با رشد اخلاقى \\
\hline
\end{tabular}


جدول ه. نتايج آزمون ركرسيون بيرامون تأثير سبكهاى فرزنديرورى و دلبستكى با رشد اخلاقى كودكان بيش از دبستان

\begin{tabular}{|c|c|c|c|c|c|c|}
\hline Sig. & $T$ & beta & SE & B & متغير & \\
\hline.$\ldots 1$ & $F / T$ &. & WIAS & VNTAF & مقدار ثابت & \multirow{4}{*}{ سبك فرزنديرورى } \\
\hline.$/ \ldots 1$ & F/Mr & & $+/ N F 9$ & $N / N+F$ & أزادكذارى & \\
\hline$\%$ & $r / h r$ &.$/ 19$ & . 1971 & $1 / M 9 \Delta$ & مستبلائه & \\
\hline.$/ \ldots 1$ & P/MY &.$/ T Y 8$ &.$/ r \Delta$ & V/egV & مقتدرائه & \\
\hline $.1 . . .1$ & NPW & 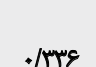 & $1 \% / \Delta 9 \Delta$ & $\pi / \pi$. & مقدار ثابت & \multirow{5}{*}{ لملبستكى } \\
\hline 1/..Y & $r / N r$ & &.$/ 919$ & $V / \Delta \Delta \mid$ & تكامل انطباقى مثبت & \\
\hline.$/ \ldots 1$ & $r / 919$ & / RAP & A & $r / q n$ & واكش هاى هيجانى & \\
\hline$\%$ & $-r / .99$ &.$- / 494$ & . $|\Delta T|$ & $-1 / 099$ & رفتارهاى مثفى & \\
\hline$\% \ldots 1$ & $-Y / R A q$ &.$- / 11 F$ & . NAS & $-1 / \cdot 11$ & دورى كزيدن از حمايت جهره دلبستكى (فرد & \\
\hline
\end{tabular}

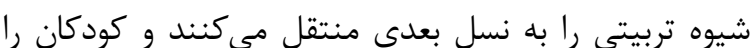

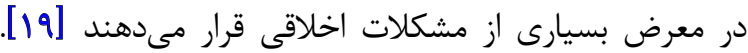

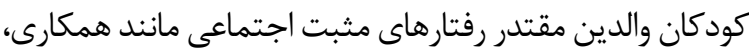

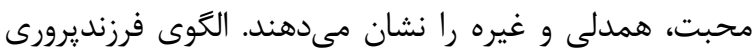

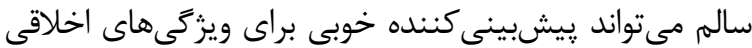

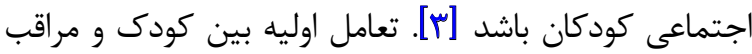

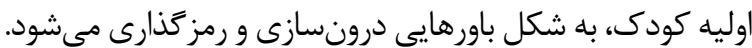

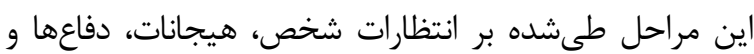

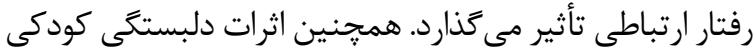

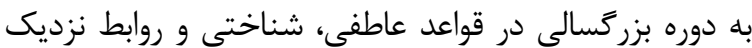

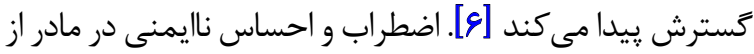

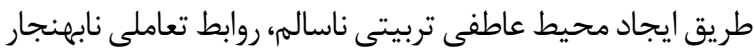

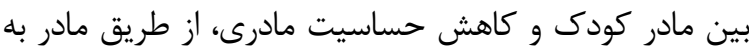

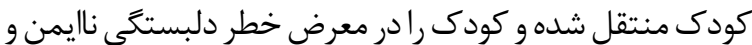

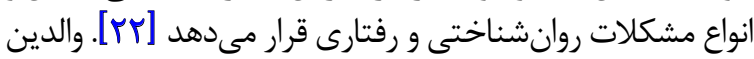

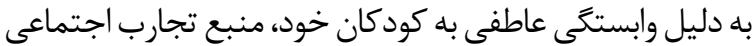

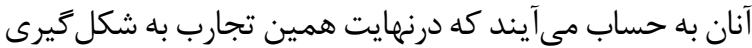

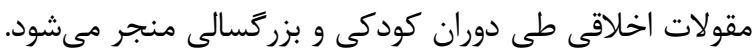

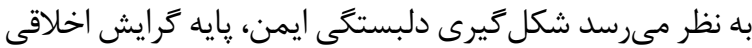

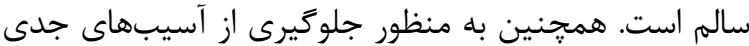

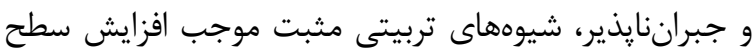
سلامت روانشناختى كودكان، رفتار آنان و سلامت روبت روان جامعه
نمره رشد اخلاقى با مؤلفههاى دلبستخى كودى رابطه معنى دارى

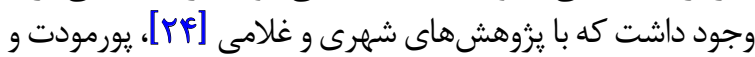

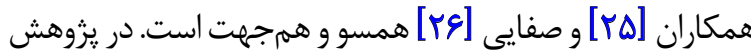

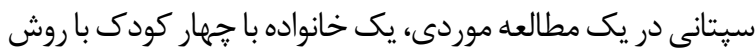

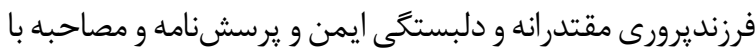

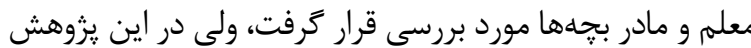

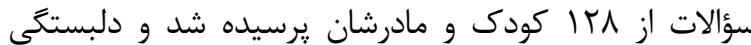

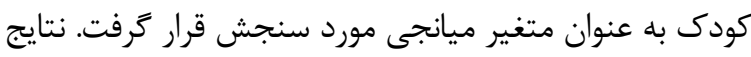

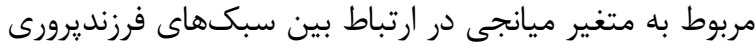

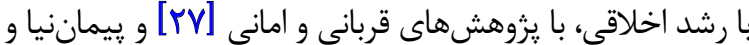

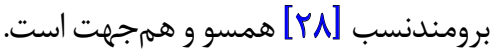

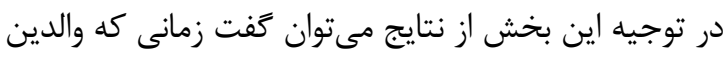

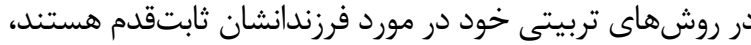

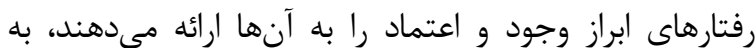

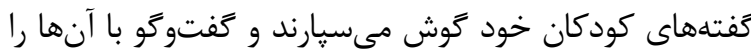

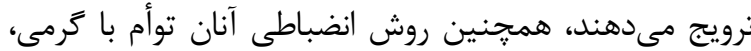

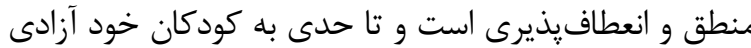

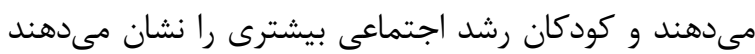

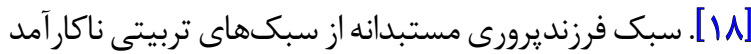

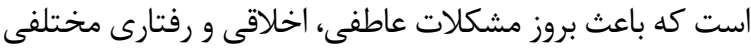

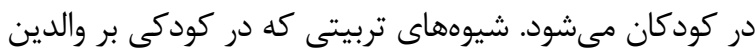

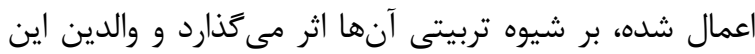

جدول 8. نتايج تحليل ميانجى مرى متغير سبكهاي دالبستكى در رابطه بين سبكهاي فرزند برورى با رشد اخلاقى

\begin{tabular}{|c|c|c|c|c|c|c|c|}
\hline Sig. & $\mathbf{R}^{2}$ & $\mathbf{R}$ & SE & $\boldsymbol{\beta}$ & $\mathbf{B}$ & مثتير وابسته & متغير مستقل \\
\hline$* \ldots$ &.$/ V F$ & I/AY & .1 .4 & I/AY &.$/ 8$. & سبكسهاى دلبستى & سبكسهاى فرزند يرورى \\
\hline $.1 \ldots$ &.$/ \pi$ &.$/ 17 q$ &.$/ M I I$ &.$/ 189$ & $1 / \pi$ & رشد اخلاقى & سبك هاى دلبسئكى \\
\hline
\end{tabular}


فرزنديرورى مقتدرانه براى ارتقاى رشد اخلاقى فرزندان مى تواند

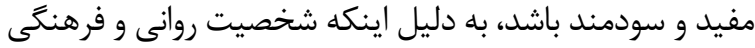

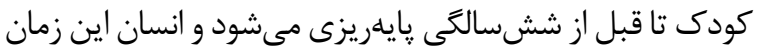

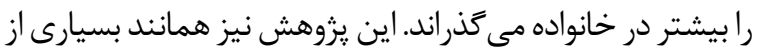

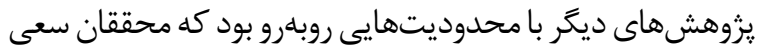

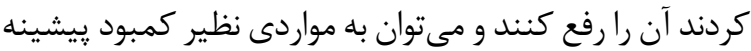

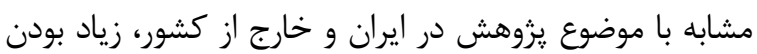

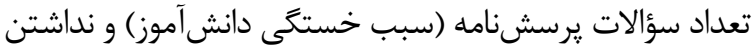

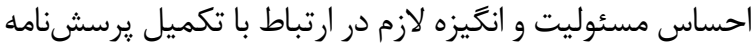
در بين برخى از مادران اشاره كرد.

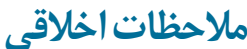

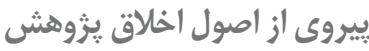

طرح يزوهشى حاضر توسط معاونت يروهشى و كميته اخلاق

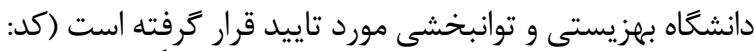

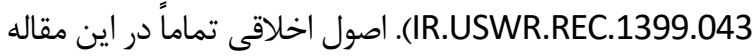

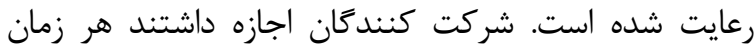

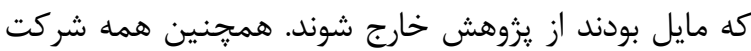

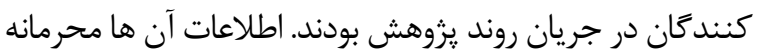

نَخه داشته شد.

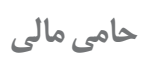

اين مقاله بركرفته از بِايان نامه كارشناسى نويسنده اول در تروه

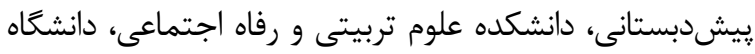
علوم توانبخشى و سلامت اجتماعى، تهران است.

$$
\text { مشار كت نويسند }
$$

مفهوم سازى: فرزانه اسمعيلى، سيامك طهماسبى، عليرضا

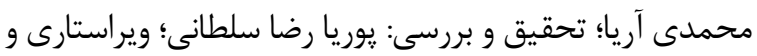

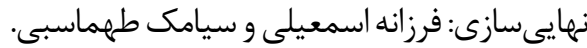

$$
\text { تعارض منافع }
$$

بنابر اظهار نويسندكان اين مقاله تعارض منافع ندارد.

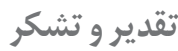

نويسند

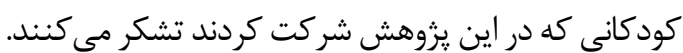

مىشود. جنان كه از نتايج اين تحقيق برمى آيد، دلبستخى در نقش إنش

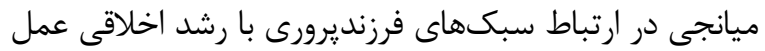

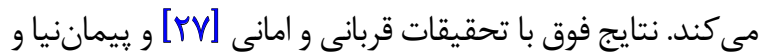

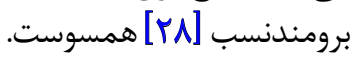

در تبيين اين يافته مىتوان كفت دلبستكى ايمن در زمان

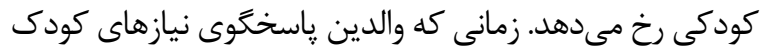

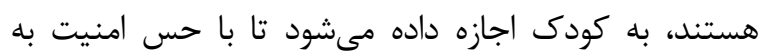

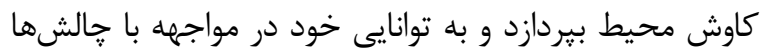

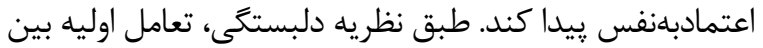

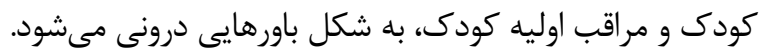

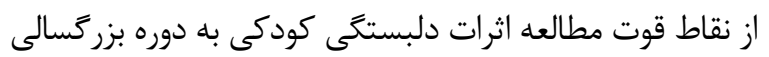

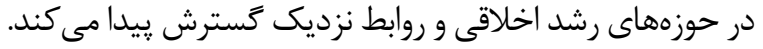

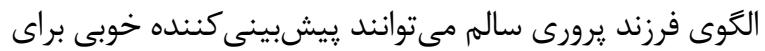

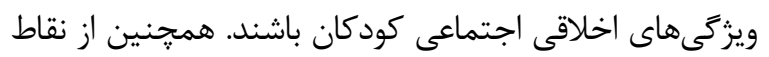

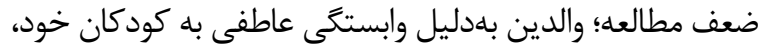

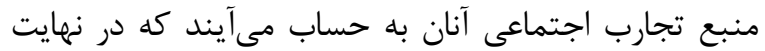

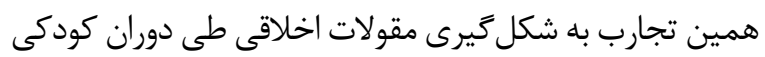

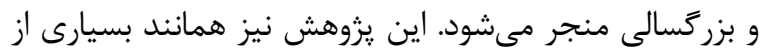

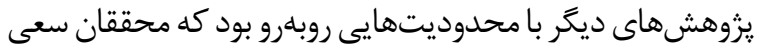

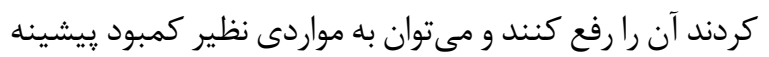

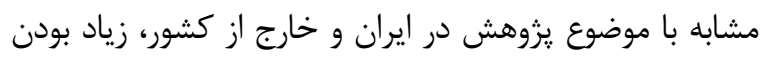

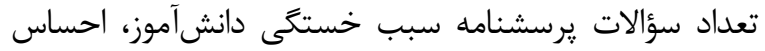

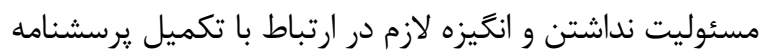
در بين برخى از مادران اشاره كرد. نتيجلَّيرى

يافتههاى يزوهش حاضر نشان داد والدين از شيوههاى

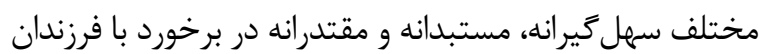

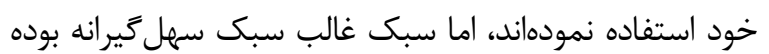

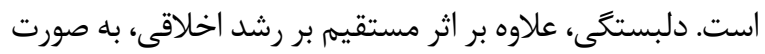

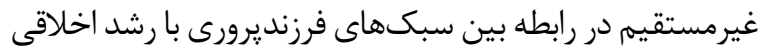

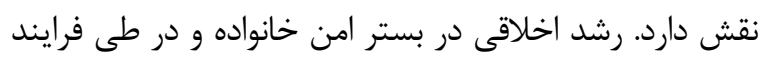

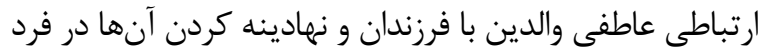

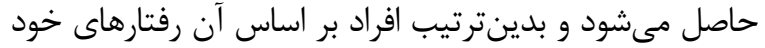

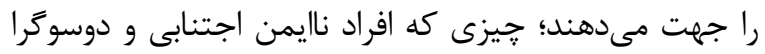

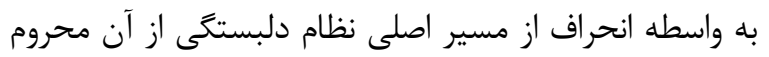

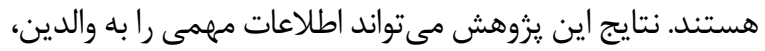

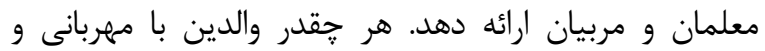

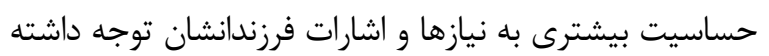

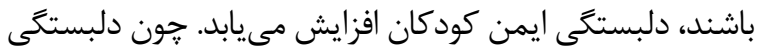

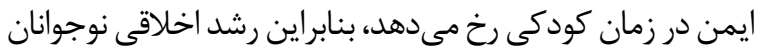

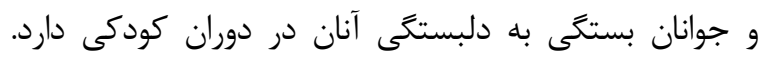

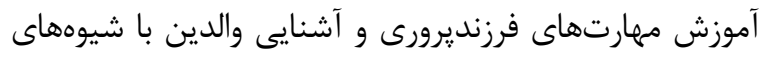




\section{References}

[1] Darvishi N. [Comparing moral growth from the perspective of Allameh Tabatabai and Kohlberg (Persian)]. Journal of Research Culture, Special for Philosophy and Theology. 2015; 8(20-21):12550. http://fpq.bou.ac.ir/article_22029.html?lang=fa

[2] Bosilki H. [Factors of children's moral development in the family (Persian)]. Revelation Ethics. 2017; 7(2):57-81. [DOI:20.1001.1.23 833025.1396.7.2.4.1]

[3] Mirzaei A. [Investigating the relationship between parenting patterns and moral and social development of third grade elementary students in amol based on Islamic perspective [MSc. thesis] (Persian)]. Sari: Mazandaran University; 2014. https://elmnet.ir/ article/10729732-93237

[4] Shoaa Kazemi M, Mir Mohammad Hossein Oshani SA, Naghib F. [The relationship between religious teachings of parents and moral development of primary school children (Persian)]. Sceintific Journal of Islamic Education. 2015; 23(29):107-20. https:// iej.ihu.ac.ir/article_200910.htmlllang=fa

[5] Jafari A, Rahmani H, Amirimajd M. [The prediction of moral judgment in talent and non-talent female students of elementary schools based on individual-family values and self- concept (Persian)]. Research in Cognitive and Behavioral Sciences. 2015; 6(2):65-80. [DOI:10.22108/CBS.2016.20995]

[6] Masoodi Alavi A, Khaleghipour S, Vahabi Homabadi J. [Structural relationship moral values according to attachment style, spiritual well-being, life orientation and among all was unsupervised adolescents: Model of high moral (Persian)]. Iranian Journal of Pediatric Nursing. 2018; 4(3):15-24. http://jpen.ir/article-1-209-en.html

[7] Janbozorgi M, Noori N, Agahheris M. [Teaching children ethics, social behavior and legality (Persian)]. Tehran: Arjmand; 2014. http://opac.nlai.ir/opac-prod/bibliographic/3638571

[8] Hosseinzadeh M, Abbasi P, Shahrokhi H, Mahdavi N, Barzanjeh Atri S. [Maternal parenting styles and its relationship with emotional-behavioral problems in children with autism spectrum disorder referred to the autism center of Tabriz university of medical sciences (Persian)]. Journal of Torbat Heydariyeh University of Medical Sciences. 2020; 7(4):11-22. http://jms.thums.ac.ir/article1-732-fa.html

[9] Azad Manesh S, Sajadieh N, Bagheri K. [The exploration of aims and considerations of child's moral education from the perspective of islamic view of action (Persian)]. Biquarterly Journal of Islamic Education. 2016; 11(23):151-73. http://islamicedu.rihu.ac.ir/article_1241_0.html?lang=en

[10] Farzadi F, Behroozi N, Shehni Yailagh M. [The causal relationship between parenting styles attachment styles and indiscipline mediated by the emotional self-regulating mediation, social skills and Theory of Mind (Persian)]. Journal of Family Psychology. 2017; 3(2):316. https://www.sid.ir/fa/Journal/ViewPaper.aspx?ID=296181

[11] Moradi Amin M, Ganji Nik M. [Approaches to child upbringing in theft by children (Persian)]. Journal of Police Cultural Studies. 2016; 3(1):133-49. magiran.com/p2230886

[12] Asakereh H, Diba H. [The role of mother in the formation of moral conscience (Persian)]. Akhlagh Religious \& Research Quarterly. 2019; 14(31):11-31. [DOI:10.22081/JARE.2019.52295.1281]
[13] Hassanpoor A, Asalani F, Goudarzi Z. [Effectiveness of emotional skills training of mothers on reducing insecure attachment and behavioral issues among elementary students in Isfahan, Iran (Persian)]. Journal of Research in Behavioural Sciences. 2016; 13(4):625-30. [DOI:20.1001.1.17352029.1394 .13.4.15.6]

[14] Devi SM, Masitoh S, Bachri BS. The role of mother-child secure attachment and mother parenting styles toward social, emotional and moral development in children aged 5-6 years old. Paper presented at: $2^{\text {nd }}$ International Conference on Education Innovation (ICEI 2018). 2018. [DOI:10.2991/ icei-18.2018.1596]

[15] Feeney JA, Karantzas GC. Couple conflict: insights from an attachment perspective. Current Opinion in Psychology. 2017; 13:60-4. [DOI:10.1016/j.copsyc.2016.04.017] [PMID]

[16] Mahasneh AM, Al-Zoubi ZH, Batayenh OT, Jawarneh MS The relationship between parenting styles and adult attachment styles from Jordan university students. International Journal of Asian Social Science. 2013; 3(6):1431-41. http:// aessweb.com/pdf-files/ijass \%203(6),\%201431-1441.pdf

[17] Rahbar S, Salari Pour E. [Determining the relationship between parenting styles and attachment with moral development in primary school students in Bandar Jask (Persian)]. Paper presened at: National Conference on Primary Education . 26-27 December 2015; Birjand, Iran. 2015;1. https:// www.sid.ir $/ \mathrm{fa} /$ seminar $/$ ViewPaper.aspx?id $=58384$

[18] Zokaeifar A, Mousazadeh T. [The role of parental parenting styles in predicting social development of preschool children 5 and 6 (Persian)]. Quarterly Social Psychology Research. 2020; 10(37):87-100. [DOI:10.22034/SPR.2020.109698]

[19] Babajan Zade Z, Khan Mohammadi O. [Investigating the relationship between parenting styles and the development of moral judgment of children 4-6 years old in Babol Kindergartens (Persian)]. Paper presented at: $2^{\text {nd }}$ International Conference on Psychology, Educational Sciences and Lifestyle. 28 January 2016; Mashhad, Iran. https://civilica.com/ doc/497623/

[20] Tarkeshdooz S, Farokhzad P. [The Relationship between children behavioral disorders with parenting styles and attachment styles (Persian)]. Journal of Community Health. 2017; 4(2):118-26. [DOI:10.22037/ch.v4i2.14372]

[21] Doodman P. [The relationship between parenting practices and accountability of high school students in Mehr Town (Persian)]. Qom University of Medical Sciences Journal. 2018; 12(4):51-60. [DOI:10.29252/qums.12.4.51]

[22] Saedi F, Khanjani Z. [A comparative study of three attachment styles (safe, ambivalent, avoidant) in adolescents with internalizing symptoms of depression and anxiety (Persian)]. Women and Family Studies. 2018; 10(38):137-54. http://jwsf. iaut.ac.ir/article_545370.html

[23] Morovati A, Vatankhah M, Morovati S. Investigating the relationship between parents' parenting styles and social development of preschool children in Ardakan in 2015-2016 (Persian)]. Paper presented at: $4^{\text {th }}$ Scientific Conference on Educational Sciences and Psychology, Social and Cultural Harms of Iran. 10 September 2016; Tehran, Iran. https:// civilica.com/doc/565568/ 
[24] Shahri K, Gholami S. [The relationship between parental attachment styles and aggression and social development of children aged 7 to 11 years (elementary school) in Behshahr (Persian)]. Paper presented at: $3^{\text {td }}$ International Conference on Psychology, Educational Sciences and Lifestyle. 25 August 2016; Mshhad, Iran. https:// civilica.com/doc/593537/

[25] Pourmavedat K, Hosseini Moghadam M, Pourmavedat M, Salah V. [Attachment styles as a precursor to aggression in preschool children (Persian)]. Paper presented at: $4^{\text {th }}$ International Conference on Recent Innovations in Psychology. 13 July 2017; Tehran, Iran. https://civilica.com/doc/670310/

[26] Safaee S. [The relationship between attachment style and social development in dyslexic and normal children (Persian)]. Journal of Exceptional Education. 2014; 14(1):29-35. http://exceptionaleducation.ir/article-1-34-en.html

[27] Ghorbani A, Amani A. [Explanatory theoretical model for parenting styles, attachment styles and differentiation of couples (persian)]. Counseling Culture and Psychotherapy. 2015; 6(21):67-86. [DOI:10.22054/QCCPC.2015.4188]

[28] Peyman Niya B, Borumand Nasab M. [The relationship between parents 'parenting styles and adolescents' moral development based on Kohlberg's moral development puzzles (Persian)]. National Congress of Child and Adolescent Psychology. 17-18 November 2015; Tehran, Iran. https://www.sid.ir/fa/seminar/ ViewPaper.aspx?ID=20506 